\title{
Effectiveness of rehabilitation for postoperative pain after surgery for spontaneous pneumothorax in young adults
}

\author{
Gaku Yamaguchi, Chimori Konaka \\ Department of Thoracic Surgery, Ichikawa Hospital, International University of Health and Welfare, Ichikawa, Japan \\ Contributions: (I) Conception and Design: G Yamaguchi; (II) Administrative support: G Yamaguchi; (III) Provision of study materials or patients: \\ Both authors; (IV) Collection and assembly of data: Both authors; (V) Data analysis and interpretation: Both authors; (VI) Manuscript writing: Both \\ authors; (VII) Final approval of manuscript: Both authors. \\ Correspondence to: Gaku Yamaguchi, MD, PhD. Department of Thoracic Surgery, Ichikawa Hospital, International University of Health and Welfare, \\ Ichikawa, Japan. Email: ugak901@gmail.com or ugaku@hotmail.com.
}

\begin{abstract}
Backgroundk Video-assisted thoracoscopic surgery (VATS) has been established as a standard therapeutic approach for pneumothorax; however, patients may experience pronounced pain during the postoperative period, and the optimization of analgesic approaches during the postoperative period is necessary. The aim of this study was to determine for the first time the effect of a physical therapist-guided exercise program on pain perception and analgesic use in the postoperative period after VATS for spontaneous pneumothorax.
\end{abstract}

Methods: This retrospective study included 73 patients aged $\leq 40$ years (mean age is 21 , range is 15 to 40 ) with spontaneous pneumothorax treated with VATS that were divided in exercise-based rehabilitation $(n=23)$ and no rehabilitation $(n=50)$ groups. Postoperative pain perception and the use of analgesics were investigated in patients who did or did not undergo an aerobic exercise postoperatively.

Results: In patients who underwent an exercise-based postoperative rehabilitation program, the pain control was more effective, the numeric rating scale (NRS) (25.46/42.31, $\mathrm{P}=0.02)$, and the dose of analgetic medication was lower than the patients who undergo rehabilitation $(\mathrm{P}=0.008)$.

Conclusions: Overall, our findings suggest for the first time that exercise-based postoperative rehabilitation may be a viable option for pain reduction after VATS for spontaneous pneumothorax in young adults.

Keywords: Pneumothorax; postoperative analgesia; postoperative pain; rehabilitation; video-assisted thoracoscopic surgery (VATS)

Submitted Aug 30, 2021. Accepted for publication Nov 16, 2021.

doi: $10.21037 /$ apm-21-1756

View this article at: https://dx.doi.org/10.21037/apm-21-1756

\section{Introduction}

Spontaneous pneumothorax represents the leakage and build-up of air between the lung and the chest wall. It may occur either without an underlying pathology or in the presence of another lung disorder (1). Spontaneous pneumothorax requires treatment, and video-assisted thoracoscopic surgery (VATS) has been established as a standard therapeutic approach (2). The use of VATS has been associated with decreased trauma, an ameliorated inflammatory response, and faster recovery than conventional open surgery $(3,4)$. Further, VATS is minimally invasive and is correlated with less postoperative pain than anterolateral thoracotomy (5).

However, patients undergoing VATS still experience marked postoperative pain in the early postoperative period (6), and the incidence of chronic postoperative pain is relatively high (7). Adequate pain control is essential to facilitate and speed up the recovery process. Different methods can be selected for postoperative analgesia, including intravenous patient-controlled opioid infusion, parenteral opioid administration, thoracic epidural analgesia, intrathecal morphine administration, intercostal 
blocks, or paravertebral opioid infusion (8). Non-steroidal anti-inflammatory drugs (NSAIDs) and paracetamol are also routinely used in the management of postoperative pain; further, $\mathrm{N}$-methyl-D-aspartate (NMDA) antagonists, dexamethasone, lidocaine, and gabapentinoids have emerged as potential therapeutic options (9).

A number of factors may influence the intensity of postoperative pain after thoracic surgery. However, only a few studies have analyzed risk factors for postoperative pain in association with VATS. Thus, in a large retrospective cohort study, the presence of moderate-to-severe VATS postoperative pain was associated with smoking history, younger age, higher body mass index (BMI), higher number of placed chest tubes, and presence of preexisting pain (10). However, the factors affecting postoperative pain after VATS should be further explored and validated.

Postoperative rehabilitation may affect postoperative pain and functional outcomes. Furthermore, the timing and type of postoperative rehabilitation should be considered and may affect functional outcomes. In patients who underwent thoracotomy for resectable lung cancer, earlier rehabilitation was associated with better exercise tolerance but stronger pain (11). However, a postoperative exercise program for the shoulders decreased pain intensity and improved function after open thoracothomy (12). Two different reports were presented and the effect of postoperative rehabilitation after VATS on pain perception has not been definitively clarified.

In this retrospective study, we assessed, for the first time, the association of physical therapist-led rehabilitation with postoperative pain in young adults (aged $\leq 40$ years) treated with VATS for spontaneous pneumothorax. Furthermore, we determined whether postoperative pain was associated with postoperative pain memory, operation history, sex, and operation duration. We present the following article in accordance with the STROBE reporting checklist (available at https://apm.amegroups.com/article/view/10.21037/apm$21-1756 / \mathrm{rc})$.

\section{Methods}

\section{Participants}

Young adults (aged $\leq 40$ years) who underwent surgery for spontaneous pneumothorax between October 1, 2012 and September 30, 2017 were included in this retrospective study. The inclusion criteria were the performance of three-port VATS and age $\leq 40$ years. The exclusion criteria were open thoracotomy ( 1 patient), cases that did not involve bullectomy (1 patient), postoperative complications expected to enhance pain (1 patient with pyothorax), and contraindications for NSAIDs (1 patient). Patients were followed up until their discharge from the hospital.

\section{Ethical aspects}

This protocol was approved by the Ethical Committee of the Ichikawa Hospital, International University of Health and Welfare (approval number: 79; date June 19, 2018). The experiments were conducted according to the World Medical Association Declaration of Helsinki (as revised in 2013). Moreover, the manuscript conforms to the ICMJE Recommendations for the Conduct, Reporting, Editing, and Publication of Scholarly Work in Medical Journals. Informed consent for the surgery and other therapeutic procedures was obtained by all patients. An opt-out procedure was used for informed consent for the study.

\section{Surgical procedure}

All patients underwent three-port VATS, and a 20 Fr chest drainage tube was placed in the thoracic cavity.

\section{Assessment of postoperative pain}

The number of times patients complained of pain was determined according to the regular nursing records. Patients described the intensity of postoperative pain using a numeric rating scale (NRS) with 11 points. A score of 0 indicated "no pain", whereas a score of 10 indicated the "worst pain imaginable" (5). Nursing staff asked 4 times on the postoperative day (POD) 0 , and 3 times on the POD 1 to POD 4. And we chose the highest numbers of the day.

\section{Postoperative analgesics use}

We measured the presence or absence of analgesic drug use other than the intravenous patient-controlled analgesia and oral loxoprofen. Our analgesic scheme included intravenous-patient controlled analgesia (IV-PCA) ( $1 \mathrm{mg}$ fentanyl in normal saline to $0.005 \mathrm{mg} / \mathrm{kg} / \mathrm{h}$ ) from the intraoperative stage to POD 1, dripping acetaminophen on POD 0, and loxoprofen administration (180 $\mathrm{mg} /$ day) from POD 1. The additional on demand drugs were IV-PCA and diclofenac administered as $25 \mathrm{mg}$ suppositories. Further, 
Table 1 Baseline characteristics of the rehabilitation and no rehabilitation groups

\begin{tabular}{|c|c|c|c|c|}
\hline Characteristics & All cases & Rehabilitation group $(n=23)$ & No rehabilitation group $(n=50)$ & $P$ value \\
\hline Days before removal of the drainage tube (mean) & 3.14 & 3.50 & 2.96 & 0.796 \\
\hline Male & 67 & 22 & 49 & 0.421 \\
\hline Age $\geq 30$ years & 16 & 4 & 12 & 0.533 \\
\hline Preoperative drainage & 42 & 15 & 27 & 0.375 \\
\hline Smoking & 20 & 7 & 13 & 0.698 \\
\hline
\end{tabular}

we assessed the frequency of analgesic use according to the regular nursing records.

\section{Rehabilitation program}

Range-of-motion exercises, walking exercises, and aerobics, but not respiratory muscle training, were instituted for the patients included in the rehabilitation group. The exercises were conducted every day except on POD 0 . They were performed in one-on-one sessions with a physical therapist, lasted for 30-40 min, and were conducted twice daily.

\section{Statistical analysis}

The data analyzed in the study were not normally distributed. Continuous or ordinal variables were compared using the Mann-Whitney U-test, whereas categorical variables were compared using the chi-square test. $\mathrm{P}$ values $<0.05$ were considered to indicate statistical significance. SPSS (version 26) was used for statistical analysis.

\section{Results}

\section{Patient baseline and clinical characteristics}

The study included 73 patients aged $\leq 40$ years that underwent surgery for spontaneous pneumothorax. The patients were divided into rehabilitation $(n=23)$ and no rehabilitation $(\mathrm{n}=50)$ groups. The patient baseline characteristics in the rehabilitation and no rehabilitation groups are presented in Table 1 . There were no significant differences between the two groups with regard to age, sex, operation duration, perioperative drainage, operation history, and smoking (Table 1).

\section{Relationship between rehabilitation and the perception of pain intensity}

The physical therapist-guided exercise rehabilitation was associated with a reduced number of pain complaints $(\mathrm{P}=0.020)$. Furthermore, rehabilitation was associated with reduced instances of the use of overall analgesics $(\mathrm{P}=0.006)$ and additional analgesics $(\mathrm{P}=0.008)$ (Table 2). An analysis of the time-course of pain intensity changes with and without rehabilitation showed that rehabilitation reduced the mean postoperative pain on Days $1(\mathrm{P}=0.03)$ and $4(\mathrm{P}=0.00)$ and mean NRS score (25.46/42.31, $\mathrm{P}=0.002)$ (Figure 1).

\section{The association of other factors with pain perception}

The association of other factors with differences in pain perception is presented in Table 2. Female sex was associated with a lower number of pain complaints overall $(\mathrm{P}=0.026)$ and on POD $0(\mathrm{P}=0.012)$. Smoking showed a tendency toward an association with a lower number of pain complaints overall $(\mathrm{P}=0.050)$ and on POD $0(\mathrm{P}=0.066)$, which did not reach statistical significance. And the sex and smoking rate did not differ between the rehabilitation and no rehabilitation groups.

\section{Discussion}

The current study identified postoperative physical therapist-guided rehabilitation as a factor associated with reduced pain perception and analgesic use during the early postoperative period. The effect of rehabilitation was observed on POD 1 and 4.

VATS is associated with considerable pain during the early postoperative period. A previous study investigated 
Table 2 Factors associated with changes in pain perception and analgesics use

\begin{tabular}{|c|c|c|c|c|c|c|c|}
\hline & $\begin{array}{c}\text { Sex } \\
\text { (female/male) }\end{array}$ & $\begin{array}{c}\text { Age } \\
(\geq 30 /<30)\end{array}$ & $\begin{array}{c}\text { Operation } \\
\text { duration } \\
(\leq 50 />50 \mathrm{~min})\end{array}$ & $\begin{array}{l}\text { Previous } \\
\text { surgery } \\
\text { (yes/no) }\end{array}$ & $\begin{array}{c}\text { Smoking } \\
\text { (has smoked/ } \\
\text { non-smoker) }\end{array}$ & $\begin{array}{l}\text { Rehabilitation } \\
\text { (yes/no) }\end{array}$ & $\begin{array}{l}\text { Drainage before } \\
\text { operation (yes/no) }\end{array}$ \\
\hline $\begin{array}{l}\text { Number of times of painkiller use } \\
\text { (POD } 0 \text { to } 9 \text { am on POD 1) }\end{array}$ & $\begin{array}{l}0.67 / 1.09 \\
(P=0.530)\end{array}$ & $\begin{array}{l}0.88 / 1.11 \\
(P=0.616)\end{array}$ & $\begin{array}{l}0.89 / 1.22 \\
(P=0.712)\end{array}$ & $\begin{array}{l}0.72 / 1.16 \\
(P=0.235)\end{array}$ & $\begin{array}{l}0.75 / 1.17 \\
(P=0.187)\end{array}$ & $\begin{array}{l}0.48 / 1.32 \\
(P=0.006)\end{array}$ & $\begin{array}{l}1.14 / 0.94 \\
(P=0.269)\end{array}$ \\
\hline $\begin{array}{l}\text { Number of times with pain } \\
\text { complaints on POD } 0\end{array}$ & $\begin{array}{l}0.50 / 2.03 \\
(P=0.012)\end{array}$ & $\begin{array}{l}1.25 / 2.09 \\
(P=0.079)\end{array}$ & $\begin{array}{l}1.67 / 2.14 \\
(P=0.262)\end{array}$ & $\begin{array}{l}1.28 / 2.11 \\
(P=0.065)\end{array}$ & $\begin{array}{l}1.30 / 2.13 \\
(P=0.066)\end{array}$ & $\begin{array}{l}1.61 / 2.04 \\
(P=0.332)\end{array}$ & $\begin{array}{l}1.50 / 2.00 \\
(P=0.418)\end{array}$ \\
\hline
\end{tabular}

POD, postoperative day.

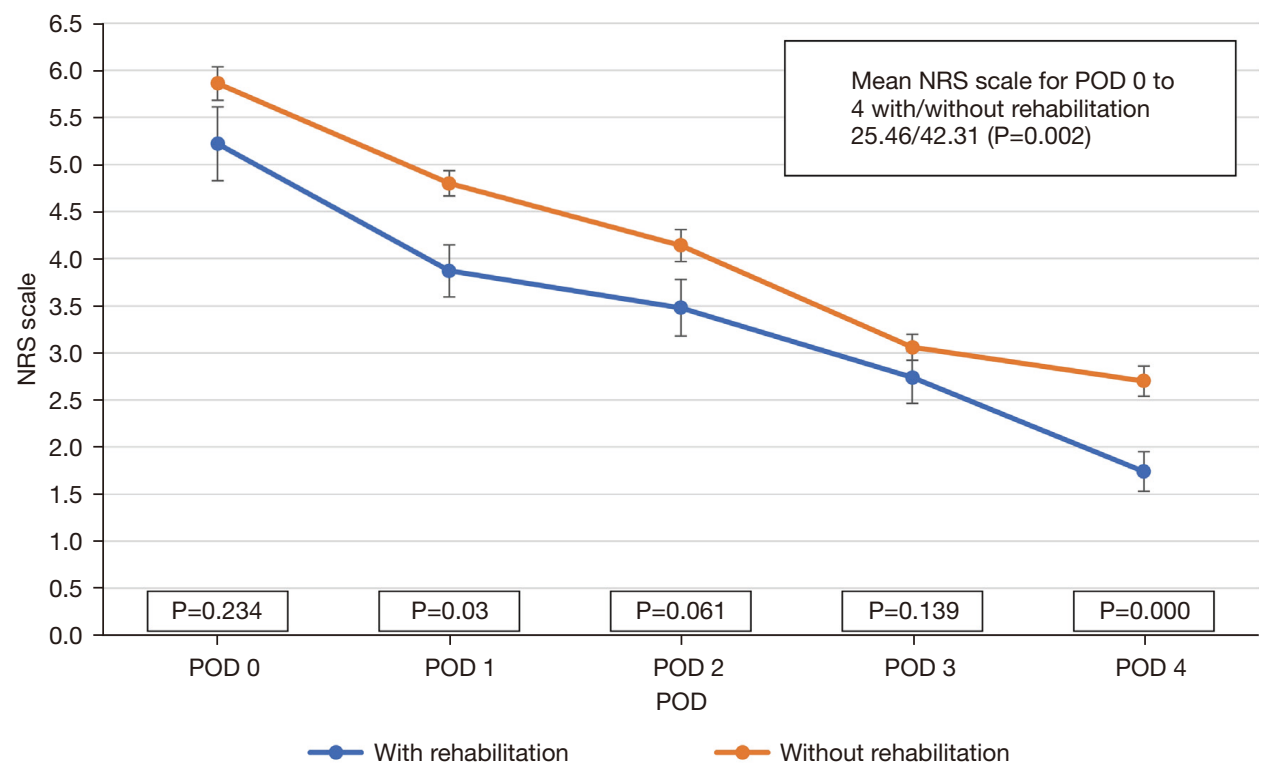

Figure 1 Relationship between rehabilitation and postoperative pain. NRS, numeric rating scale; POD, postoperative day.

the factors that correlated with patient satisfaction of pain management (13). The possibility to participate in decisions regarding pain management and the access to helpful information regarding therapeutic options for pain were positively associated with patient satisfaction of pain management. Conversely, the pain intensity level at the time of assessment, the detection of the lowest pain score in the preceding $24 \mathrm{~h}$, and the interference of postoperative pain with sleep were negatively associated with patient satisfaction of pain management (13). However, other factors may also be associated with adequate pain control and with the amount of analgesics used during the postoperative period.

Physiotherapy-based rehabilitation can be an important part of the multimodal postoperative care after thoracic surgery to achieve optimal recovery. The physiotherapy plan is based on an initial assessment and may include early mobilization in addition to the pain management, positioning, airway clearance, and lung expansion techniques (14). The type and timing of the physiotherapy exercises should follow scientific evidence; however, knowledge regarding the influence of physiotherapybased rehabilitation on postoperative outcomes in patients undergoing VATS is limited. 
To the best of our knowledge, our study demonstrates, for the first time, an association of VATS postoperative physiotherapy rehabilitation with reduced pain perception and analgesics use. A previous study found that relaxation exercises were associated with reduced postoperative pain in patients undergoing upper abdominal surgery (15). Moreover, Danielsen et al. (16) showed that in patients undergoing discectomy for lumbar disc herniation, postoperative medical exercise therapy decreased pain and disability without compromising clinical outcomes. As mentioned earlier, a postoperative exercise program for the shoulders decreased the intensity of postoperative pain, including shoulder pain, and improved function after open thoracotomy (12). Our study further substantiates previous findings on the utility of exercise programs for postoperative pain reduction and provides specific information for patients who had undergone VATS.

In our study, none of the other assessed demographic and clinical factors besides rehabilitation affected the amount of analgesics used. However, female sex and smoking habit was also associated with a lower number of pain complaints $(\mathrm{P}=0.026$ and $\mathrm{P}=0.050)$. But the number of females and smoking patient were so small because the spontaneous pneumothorax tends to occur on males and minors.

The main limitation of our study is its retrospective design. In the future, prospective randomized controlled trials should be conducted to validate these findings. Moreover, our study was conducted at a single center, and it should be replicated in other settings to allow generalization of these findings.

Overall, our findings suggest that rehabilitation may be a viable option for pain reduction after VATS for spontaneous pneumothorax in young adults.

\section{Acknowledgments}

We would like to thank Y Fukawa and S Yoshida for their help with the physical therapy of the patients. Editorial support, in the form of medical writing, assembling tables and creating high-resolution images based on authors' detailed directions, collating author comments, copyediting, fact checking, and referencing, was provided by Editage, Cactus Communications.

Funding: None.

\section{Footnote}

Reporting Checklist: The authors have completed the
STROBE reporting checklist. Available at https://apm. amegroups.com/article/view/10.21037/apm-21-1756/rc

Data Sharing Statement: Available at https://apm.amegroups. com/article/view/10.21037/apm-21-1756/dss

Peer Review File: Available at https://apm.amegroups.com/ article/view/10.21037/apm-21-1756/prf

Conflicts of Interest: Both authors have completed the ICMJE uniform disclosure form (available at https://apm. amegroups.com/article/view/10.21037/apm-21-1756/coif). The authors have no conflicts of interest to declare.

Ethical Statement: The authors are accountable for all aspects of the work in ensuring that questions related to the accuracy or integrity of any part of the work are appropriately investigated and resolved. This protocol was approved by the Ethical Committee of the Ichikawa Hospital, International University of Health and Welfare (approval number: 79; date June 19, 2018). The experiments were conducted according to the World Medical Association Declaration of Helsinki (as revised in 2013). Moreover, the manuscript conforms to the ICMJE Recommendations for the Conduct, Reporting, Editing, and Publication of Scholarly Work in Medical Journals. Informed consent for the surgery and other therapeutic procedures was obtained by all patients. An opt-out procedure was used for informed consent for the study.

Open Access Statement: This is an Open Access article distributed in accordance with the Creative Commons Attribution-NonCommercial-NoDerivs 4.0 International License (CC BY-NC-ND 4.0), which permits the noncommercial replication and distribution of the article with the strict proviso that no changes or edits are made and the original work is properly cited (including links to both the formal publication through the relevant DOI and the license). See: https://creativecommons.org/licenses/by-nc-nd/4.0/.

\section{References}

1. Zarogoulidis P, Kioumis I, Pitsiou G, et al. Pneumothorax: from definition to diagnosis and treatment. J Thorac Dis 2014;6:S372-6.

2. Al-Mourgi M, Alshehri F. Video-Assisted Thoracoscopic Surgery for the Treatment of First-Time Spontaneous Pneumothorax versus Conservative Treatment. Int J 
Health Sci (Qassim) 2015;9:428-32.

3. Yim AP. Minimizing chest wall trauma in video-assisted thoracic surgery. J Thorac Cardiovasc Surg 1995;109:1255-6.

4. Yim AP, Wan S, Lee TW, et al. VATS lobectomy reduces cytokine responses compared with conventional surgery. Ann Thorac Surg 2000;70:243-7.

5. Bendixen M, Jørgensen OD, Kronborg C, et al. Postoperative pain and quality of life after lobectomy via video-assisted thoracoscopic surgery or anterolateral thoracotomy for early stage lung cancer: a randomised controlled trial. Lancet Oncol 2016;17:836-44.

6. Tamura M, Shimizu Y, Hashizume Y. Pain following thoracoscopic surgery: retrospective analysis between single-incision and three-port video-assisted thoracoscopic surgery. J Cardiothorac Surg 2013;8:153.

7. Passlick B, Born C, Sienel W, et al. Incidence of chronic pain after minimal-invasive surgery for spontaneous pneumothorax. Eur J Cardiothorac Surg 2001;19:355-8; discussion 358-9.

8. Allain PA, Carella M, Agrafiotis AC, et al. Comparison of several methods for pain management after video-assisted thoracic surgery for pneumothorax: an observational study. BMC Anesthesiol 2019;19:120.

9. Piccioni F, Segat M, Falini S, et al. Enhanced recovery pathways in thoracic surgery from Italian VATS Group: perioperative analgesia protocols. J Thorac Dis

Cite this article as: Yamaguchi G, Konaka C. Effectiveness of rehabilitation for postoperative pain after surgery for spontaneous pneumothorax in young adults. Ann Palliat Med 2022;11(4):1191-1196. doi: 10.21037/apm-21-1756
2018;10:S555-63.

10. Sun K, Liu D, Chen J, et al. Moderate-severe postoperative pain in patients undergoing video-assisted thoracoscopic surgery: A retrospective study. Sci Rep 2020;10:795.

11. Stigt JA, Uil SM, van Riesen SJ, et al. A randomized controlled trial of postthoracotomy pulmonary rehabilitation in patients with resectable lung cancer. J Thorac Oncol 2013;8:214-21.

12. Reeve J, Stiller K, Nicol K, et al. A postoperative shoulder exercise program improves function and decreases pain following open thoracotomy: a randomised trial. J Physiother 2010;56:245-52.

13. Siu E, Quick JS, Xu X, et al. Evaluation of the Determinants of Satisfaction With Postoperative Pain Control After Thoracoscopic Surgery: A Single-Center, Survey-Based Study. Anesth Analg 2019;128:555-62.

14. Ahmad AM. Essentials of Physiotherapy after Thoracic Surgery: What Physiotherapists Need to Know. A Narrative Review. Korean J Thorac Cardiovasc Surg 2018;51:293-307.

15. Topcu SY, Findik UY. Effect of relaxation exercises on controlling postoperative pain. Pain Manag Nurs 2012;13:11-7.

16. Danielsen JM, Johnsen R, Kibsgaard SK, et al. Early aggressive exercise for postoperative rehabilitation after discectomy. Spine (Phila Pa 1976) 2000;25:1015-20. 